\title{
Risk prediction for candidemia in surgical intensive care unit patients
}

\author{
(1) Aysegul Ulu Kilic, ${ }^{1}$ (i) Sare Merve Basaga, ${ }^{1}$ (i) Fatma Cevahir, $^{2}$ (i) Ozlem Cakir, ${ }^{2}$ (i) Mehmet Doganay, ${ }^{1}$ \\ (D) Emine Alp 3 \\ ${ }^{1}$ Department of Infectious Diseases, Erciyes University Faculty of Medicine, Kayseri, Turkey \\ ${ }^{2}$ Infection Control Committee, Erciyes University Faculty of Medicine, Kayseri, Turkey \\ ${ }^{3}$ Turkish Ministry of Health, Ankara, Turkey
}

\begin{abstract}
OBJECTIVE: Patients in surgical intensive care units are thought to be at the highest risk for developing candidemia, especially patients undergoing abdominal surgery. The present study aims to investigate risk factors for candidemia in patients with abdominal surgery.
\end{abstract}

METHODS: A retrospective study was undertaken that involved patients admitted to the surgical ICU between January 2016 and January 2017. All postoperative adult patients (>18 years old) who underwent abdominal surgery were included in this study.

RESULTS: During the one-year study period, 49 patients developed candidemia. Thirty-five of candida isolates were non-albicans strains. Of them, $25(51 \%)$ isolates were Candida parapsilosis, eight (16.3\%) isolates were C. glabrata, one (2\%) isolate was $C$. tropicalis and one (2\%) isolate was $C$. kefyr. The median age of all patients enrolled in this study was $60.5 \pm 15.6$ years. In univariate analysis, the duration of the hospital stays, intensive care unit stay, type of surgery, respiratory failure, total parenteral nutrition, transfusion and use of central venous catheter were significantly higher in patients with candidemia. In multivariate analysis, duration of hospital and intensive care unit stay and use of central venous catheter was associated with an increased risk of candidemia. The mortality rate of case patients was $36.7 \%$.

CONCLUSION: Patients undergoing abdominal surgery are at increased risk of candidemia, especially the patients with prolonged intensive care unit/hospital stay and the patients with a central venous catheters. Antifungal prophylaxis may be considered for patients with increased risk.

Keywords: Candidemia; intensive care unit; surgery.

Cite this article as: Ulu Kilic A, Basaga SM, Cevahir F, Cakir O, Doganay M, Alp E. Risk prediction for candidemia in surgical intensive care unit patients. North Clin Istanb 2020;7(4):348-353.

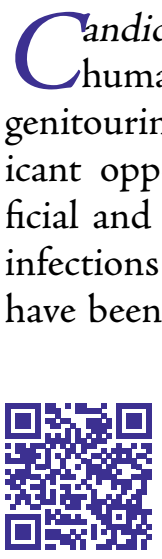

A point prevalence survey of health care-associated infections carried out in acute care hospitals from several states of USA revealed that Candida species are found to be the first common bloodstream infection in the U.S. acute care hospitals [2]. Furthermore, during recent decades, non-albicans species have increased significantly and accounted for more than half of all candidemia cases. 
The incidence and prevalence of candidemia are higher among immunocompromised and/or patients hospitalized with serious underlying disease in intensive care units. In addition, mortality attributed to candidemia is high as $47 \%$ among adults and $29 \%$ among children [3].

The risk for developing candidemia is higher among patients hospitalized in SICU, especially the patients undergoing abdominal surgery. Various risk factors previously reported associated with invasive candidiasis in SICU patients depending on the diverse nature of the patient populations and hospitals $[4,5]$. Given the high morbidity and mortality of the disease, antifungal prophylaxis (AP) for high-risk patients has been recommended previously, but the role of this approach is still remaining controversial $[5,6]$. A recent meta-analysis of randomized placebo trials revealed that prophylaxis reduced the fungal infections, but not associated mortality among high-risk surgical ICU patients [5-7]. On the other hand, the clinicians have a dilemma to face a shift to less susceptible or resistant non-albicans species due to azole prophylaxis $[6,7]$. To identify risk factors for who will benefit most from AP should be based on the local epidemiological data and patient characteristics. In this study, we aimed to investigate the patients at high risk of candidemia among whom undergoing abdominal surgery.

\section{MATERIALS AND METHODS}

\section{Patients and settings}

The current study was carried out in a University Hospital located in the Central Anatolia region. It is a tertiary referral hospital with a total capacity of 1300 -beds. The SICU is a 12 -bed adult critical care unit providing complex care to patients with abdominal surgery. The general surgery ward has 72 inpatient beds. Postoperative patients are frequently hospitalized in ICU (duration differs according to the severity of illness of the patient), then transferred to ward if clinically stable.

\section{Study Design and Data Collection}

A retrospective study was undertaken that involved all postoperative adult patients ( $>18$ years) who underwent abdominal surgery and hospitalized in surgical ICU between January 2016 and January 2017. Case patients were defined as the cases with the culture of a blood specimen yielded a Candida species collected $48 \mathrm{~h}$ after admission to the SICU. Control group comprised patients who underwent abdominal surgery but did not have any infection or colonization with Candida spp. during the hospital stay.

Standardized surveillance definitions of health care infection definitions were used for bloodstream infection due to Candida spp. [8]. The first episode of each patient with Candida BSI was included in the analysis.

The data collection used for risk factor analysis continued until candidemia developed for case patients, whereas for controls, for the total duration of the stay in the SICU. Obtained data included demographics, length of stay, underlying diseases and invasive procedures.

Major surgery is defined as any invasive operative procedure in which a more extensive resection is performed (e.g., a body cavity is entered, organs are removed, or normal anatomy is altered). Laparoscopic or open repair or resection of the stomach, small bowel, colon, liver, pancreas, spleen, adrenals or liver and open cholecystectomy was described as major surgery. Minor surgeries included laparoscopic cholecystectomy and endoscopic procedures. Inguinal or umbilical hernia repair by laparoscopic or open approach was also included in this group. This category also includes biopsy, an invasive operative procedure for the procurement of tissue samples.

Anastomotic leakage defined as a defect of the intestinal wall at the anastomotic site (including suture and staple lines of neorectal reservoirs) causing a communication between the intra- and extraluminal compartments [9].

\section{Identification and Antifungal Susceptibility}

BACTEC 9240 blood culture system (Becton Dickinson, Cockeysville, MD, USA) was used for blood specimens from patients with suspected candidemia. After the automated alert system, signal of a positive blood culture subcultures were performed from blood culture bottles to CHROMagar Candida (BBL France) and Sabouraud dextrose agar (Oxoid, United Kingdom). The identification of the Candida spp. were addressed through conventional methods, including microscopic morphologic examinations and biochemical tests and API 20C AUX (Biomerieux, France).

The antifungal susceptibility of Candida species isolated from blood specimens was evaluated by Clinical and Laboratory Standards Institute (CLSI) M27-A3 standard using microdilution (voriconazole, amphotericin B, fluconazole) and E-test (AB Biodisc, Switzerland) (caspofungin) methods. 
TABLE 1. Operative procedures of the patients included in this study

\begin{tabular}{lcc} 
Operative procedures & $\begin{array}{c}\text { Cases } \\
\mathrm{n}=49, \%\end{array}$ & $\begin{array}{c}\text { Controls } \\
\mathrm{n}=136, \%\end{array}$ \\
\hline Small bowel surgery & 32.7 & 17.6 \\
Gastric surgery & 24.5 & 11.8 \\
Bile duct, liver or pancreatic surgery & 12.2 & 14.7 \\
Colon surgery & 22.4 & 36.8 \\
Gallbladder surgery & 4.1 & 14.7 \\
Pancreaticoduodenectomy & & \\
(Whipple Procedure) & 2.0 & 0.0 \\
Diaphragmatic hernia repair & 2.0 & 0.0
\end{tabular}

\section{Algorithm for Diagnosis and Treatment of IC}

The $(1,3)$ B-D-glucan assay was not readily available in SICUs during the study period. A bedside approach (Candida score) for the empirical use of antifungals in patients with suspected IC is generally preferred in ICUs. Echinocandins are usually used for first-line therapy for moderate to severely ill patients or in patients with previous exposure to azoles. For targeted therapy of IC, the initial choice is often with echinocandins and transition to fluconazole done after confirmation of susceptibility. Fluconazole prophylaxis against IC is not routinely practiced for patients in SICU.

\section{Statistical Analysis}

SPSS software version 15 (USA) was performed for statistical analysis of all data in this study. The chi-square test was used to determine the association between the categorical variables. To compare the case and control groups, the Mann-Whitney $U$ test was used to determine the differences between continuous variables. Univariate and multiple binary logistic regression analyses (model: backward Wald) were performed to analyze the effects of variables. The level of significance was set at $\mathrm{p}<0.05$ for all tests.

\section{Ethics}

The authors confirm that ethical approval was received from the Non-invasive Clinical Research Ethics Committee of Erciyes University (date: 24.07.2019, number: 2019/579).

\section{RESULTS}

A total of 185 patients underwent abdominal surgery during the study period. The operative procedures of patients are listed in Table 1 . The median age of all patients enrolled was $60.5 \pm 15.6$ years. Of all, 49 (26.4\%) patients had developed candidemia. Thirty-five of candi$d a$ isolates were non-albicans strains. Of them, 25 (51\%) were Candida parapsilosis, eight (16.3\%) patients were Candida glabrata, one (2\%) patient was Candida tropicalis and one (2\%) patient was Candida kefyr. In vitro antifungal susceptibility results revealed that fluconazole resistance among Candida spp. was 4/49 (8.2\%).

In univariate analysis, duration of hospital stays, ICU stay, type of surgery, respiratory failure, total parenteral nutrition, transfusion and use of central venous catheter were significantly higher in patients with candidemia. The duration of ICU stay of patients with candidemia was 15.5 days (3-110) and was significantly longer than controls $(p=0.001)$ In multivariate analysis, duration of hospital stay and "to be in ICU for more than two days" and use of CVC was associated with increased risk of candidemia (Table 2).

The mortality rate of case patients was $36.7 \%$. Of all, $80 \%$ of the patients received antifungal therapy; $66 \%$ of the patients were treated with echinocandin, while $34 \%$ of the patients were treated with fluconazole. The patients received antifungal therapy within three days (median) after diagnosis. The median duration of antifungal therapy was 11 (2-28) days (Table 3).

\section{DISCUSSION}

Multiple risk factors found to be associated with the increased risk of candidemia, including parenteral nutrition, usage of wide-spectrum antibiotics and central line catheters [1-4]. Abdominal surgery brings about a predisposition for developing candidemia, both altering the gastrointestinal tract flora and damage the natural mucosal barriers. As compared with the other ICUs, patients hospitalized in surgical ICU are also found at higher risk for candidemia [4]. Therefore, it is crucial for clinicians to be aware of risk factors among this subgroup of patients who are at high risk for candidemia.

Prophylaxis against invasive candida infections for surgical patients who were at increased risk was argued previously $[6,7]$. Given the high mortality of candidemia, fluconazole prophylaxis seems to be an appropriate approach for patients at risk. A meta-analysis of randomized, placebo-controlled trials revealed the decreased rates of invasive candidiasis of high-risk surgical patients with the use of AP [7]. Selecting the candidate 
TABLE2. Univariate and multivariate analysis of risk factors for candidemia in patients undergoing abdominal surgery

\begin{tabular}{|c|c|c|c|c|c|}
\hline \multirow[t]{2}{*}{ Variables } & \multirow[t]{2}{*}{ Cases $n=49$} & \multirow[t]{2}{*}{ Controls $n=136$} & \multirow[t]{2}{*}{$\mathrm{p}$} & \multicolumn{2}{|c|}{ Multivariate analysis } \\
\hline & & & & OR & $\mathrm{p}$ \\
\hline Mean age $\pm S D$ & $62.7 \pm 14.7$ & $59.7 \pm 16.3$ & 0.270 & & \\
\hline Male gender & $27(55.1)$ & $75(55.1)$ & 0.996 & & \\
\hline Comorbidity, median & 6 & 7 & 0.442 & & \\
\hline Duration of total stay, median & 24 & 10 & 0.001 & 1.07 & 0.001 \\
\hline Time up to the development of the infection, median & 10 & 6 & 0.080 & & \\
\hline ICU stay (>2 days) $(\%)$ & 93.9 & 55.9 & 0.001 & 6.17 & 0.028 \\
\hline Anastomotic leak (\%) & $10 / 34(29.4)$ & $15 / 115(13.0)$ & 0.025 & & \\
\hline \multicolumn{6}{|l|}{ Type of surgical (\%) } \\
\hline Major & 98.0 & 86.0 & 0.028 & & \\
\hline Minor & 2.0 & 14.0 & & & \\
\hline \multicolumn{6}{|l|}{ Underlying diseases and conditions (\%) } \\
\hline Malignancy & 44.9 & 45.6 & 0.934 & & \\
\hline Diabetes mellitus & 16.3 & 19.1 & 0.665 & & \\
\hline Hypertension & 16.3 & 21.3 & 0.453 & & \\
\hline COPD & 8.2 & 5.1 & 0.485 & & \\
\hline CAD & 18.4 & 10.3 & 0.142 & & \\
\hline Renal failure & 4.1 & 3.7 & 1.000 & & \\
\hline Respiratory failure & 34.7 & 12.5 & 0.001 & & \\
\hline Previous use of antibiotics (\%) & 44.9 & 38.6 & 0.541 & & \\
\hline \multicolumn{6}{|l|}{ Invasive devices and procedures (\%) } \\
\hline Total parenteral nutrition & 55.1 & 36.0 & 0.020 & & \\
\hline Enteral nutrition & 18.4 & 7.4 & 0.029 & & \\
\hline Chest tube & 4.1 & 1.5 & 0.575 & & \\
\hline Transfusion & 61.2 & 25.7 & 0.001 & & \\
\hline Arterial catheter & 22.4 & 15.4 & 0.266 & & \\
\hline Urinary catheter & 93.9 & 97.1 & 0.317 & & \\
\hline Hemodialysis & 14.3 & 7.4 & 0.150 & & \\
\hline Intubation/mechanical ventilation & 49.0 & 21.3 & 0.001 & & \\
\hline Tracheostomy & 10.2 & 0.7 & 0.005 & & \\
\hline CVC & 71.4 & 28.7 & 0.001 & 2.98 & 0.036 \\
\hline Drainage catheter & 67.3 & 77.2 & 0.174 & & \\
\hline Nasogastric drainage & 46.9 & 35.3 & 0.151 & & \\
\hline Colostomy/Ileostomy & 16.3 & 19.9 & 0.589 & & \\
\hline
\end{tabular}

OR: Odd ratios; SD: Standard deviation; COPD: Chronic obstructive pulmonary disease; CAD: Coronary artery disease; CVC: Central venous catheter.

patients for prophylaxis may be beneficial according to the local epidemiological characteristics of cases and resistance patterns. Since the success of AP in trials may vary according to the specific patient groups selected (e.g., transplant patients) distribution and sensitivity pattern of Candida species, the antifungal agent used, dose and duration of these agents.
In this current study, almost one-fourth of patients developed candidemia among all underwent abdominal surgery. The risk of candidemia found to be significantly associated with prolonged hospital and ICU stay ( $>2$ days). This may be clearly explained by that patients, especially with longer hospital/ICU stay, are more likely to be extended-spectrum antibiotics, which may result 
TABLE 3. Mortality rates, Candida species isolated and use of antifungals in patients with candidemia

Variables

Cases

$(n=49)$

Thirty-day mortality of patients

with candidemia (\%)

Use of antifungals for the treatment

of candidemia cases (\%)

79.6

Fluconazole

26.5

Echinocandins

53.1

Initiation of antifungals after candidemia, median day (min.-max.)

Median duration of antifungals

$3[(-11)-18]$

Species (\%)

Candida spp (not identified)

$11(2-29)$

C. parapsilosis

6.1

C. albicans

51.0

C. glabrata

22.4

C. tropicalis

16.3

C. kefyr

Min.: Minimum; Max.: Maximum.

in candida superinfection. Also, administration of TPN and use of intravenous catheters are additional risk factors that tend to increase the frequency of candida colonization during the hospitalization course in ICU. The combination of these risk factors contributes to the worse outcomes in patients.

The risk of shift to non-albicans infections and the resistance is the major concern of azole prophylaxis. Previous studies evaluated examining whether there is an association between fluconazole prophylaxis, particularly for those who did not used the selection-based method for which the patient is appropriate for prophylaxis and the distribution of Candida species. This may play a role in selection pressure among patients and an increase of infection with non-albicans strains [10]. In this study, the highest proportion responsible for the shift of non-albicans candidemia was due to Candida parapsilosis. Appropriate infection control measures to prevent catheter-related infections should be prioritized since the presence of a central venous line was found as a significant risk factor for candidemia. C. parapsilosis also may indicate geographic compatibility as it is the second most common species in southern Europe and Brazil [11]. In this study, 2 of the 49 candida strains were found to be resistant to fluconazole and two other found to be dose depended sensitive; however, resistant strains were ranked in $C$. glabrata and albicans.

The economic aspect is another impediment for prophylaxis, particularly if the selection of cases is not appropriately designed. AP for the specified group of patients who underwent abdominal surgery would be economically beneficial when compared to give all SICU patients or treating with expensive antifungal treatments, including new echinocandines [12]. Likewise, a meta-analysis found that fluconazole did not statistically alter the rate of candidemia and concluded that trials should focus on more specifically identifying patients at high risk for fungal infections [13]. This is a preliminary study carried out for precisely identifying patients at high risk for candidemia and choosing the candidate patients who will benefit from fluconazole prophylaxis. We also analyzed the distribution of Candida spp. isolates and the rate of fluconazole resistance in patients underwent abdominal surgery.

This study was in a retrospective design may be a limitation causing missing data. We could not reach full data for anastomotic leakage and also the data for APACHE II scores of patients, which are important to evaluate the severity of illness for fatal cases.

Early identifying the patients at risk and initiation of empirical therapy may be an alternative approach for high-risk surgical patients. In this current study, antifungal treatment initiated with a median of three days (up to 18 days) for patients who underwent abdominal surgery. However, the thirty-day mortality was found very high, approximately $36 \%$, which may be reduced by early recognizing of the high-risk patients. Median duration of the antifungal therapy was found shorter than recommended in guidelines (11 days) because 18 (37\%) patients died while they are receiving antifungal therapy.

Invasive candidiasis, including candidemia, is a significant problem in SICUs, solutions are needed for specific subgroups. Patients undergoing abdominal surgery are at increased risk of candidemia, especially the patients with prolonged ICU or hospital stay and the patients with CVC catheter. Institutions should describe their specific groups of patients and risk factors. AP may be conceivably considered for patients with increased risk.

Ethics Committee Approval: The authors confirm that ethical approval was received from the Non-invasive Clinical Research Ethics Committee of Erciyes University (date: 24.07.2019, number: 2019/579). 
Conflict of Interest: No conflict of interest was declared by the authors.

Financial Disclosure: The authors declared that this study has received no financial support.

Authorship Contributions: Concept - AUK; Design - AUK; Supervision - AUK, EA, MD; Fundings - AUK, EA; Materials - AUK; Data collection and/or processing - SMB, FC, OC; Analysis and/or interpretation - FC; Literature review - AUK; Writing - AUK; Critical review - AUK, MD.

\section{REFERENCES}

1. Sardi JCO, Scorzoni L, Bernardi T, Fusco-Almeida AM, Mendes Giannini MJS. Candida species: current epidemiology, pathogenicity, biofilm formation, natural antifungal products and new therapeutic options. J Med Microbiol 2013;62:10-24. [CrossRef]

2. Magill SS, Edwards JR, Bamberg W, Beldavs ZG, Dumyati G, Kainer MA, et al; Emerging Infections Program Healthcare-Associated Infections and Antimicrobial Use Prevalence Survey Team. Multistate point-prevalence survey of health care-associated infections. $\mathrm{N}$ Engl J Med 2014;370:1198-208. [CrossRef]

3. Pfaller MA, Andes DR, Diekema DJ, Horn DL, Reboli AC, Rotstein $\mathrm{C}$, et al. Epidemiology and outcomes of invasive candidiasis due to non-albicans species of Candida in 2,496 patients: data from the Prospective Antifungal Therapy (PATH) registry 2004-2008. PLoS One 2014;9:e101510. [CrossRef]

4. Ulu Kilic A, Alp E, Cevahir F, Ture Z, Yozgat N. Epidemiology and cost implications of candidemia, a 6-year analysis from a developing country. Mycoses 2017;60:198-203. [CrossRef]

5. Orsetti E, Brescini L, Mazzanti S, Trave F, Morroni G, Masucci A, et al. Characterisation of candidemia in patients with recent surgery: $A$ 7-year experience. Mycoses 2019;62:1056-63. [CrossRef]

6. Calandra T, Marchetti O. Clinical trials of antifungal prophylaxis among patients undergoing surgery. Clin Infect Dis 2004;39 Suppl 4:S185-92. [CrossRef]

7. Vardakas KZ, Samonis G, Michalopoulos A, Soteriades ES, Falagas ME. Antifungal prophylaxis with azoles in high-risk, surgical intensive care unit patients: a meta-analysis of randomized, placebo-controlled trials. Crit Care Med 2006;34:1216-24. [CrossRef]

8. Horan TC, Andrus M, Dudeck MA. CDC/NHSN surveillance definition of health care-associated infection and criteria for specific types of infections in the acute care setting [published correction appears in Am J Infect Control. 2008 Nov;36(9):655]. Am J Infect Control 2008;36:309-32. [CrossRef]

9. Rahbari NN, Weitz J, Hohenberger W, Heald RJ, Moran B, Ulrich A, et al. Definition and grading of anastomotic leakage following anterior resection of the rectum: a proposal by the International Study Group of Rectal Cancer. Surgery 2010;147:339-51. [CrossRef]

10. Zilberberg M, Yu HT, Chaudhari P, Emons MF, Khandelwal N, Shorr AF. Relationship of fluconazole prophylaxis with fungal microbiology in hospitalized intra-abdominal surgery patients: a descriptive cohort study. Crit Care 2014;18:590. [CrossRef]

11. Guinea J. Global trends in the distribution of Candida species causing candidemia. Clin Microbiol Infect 2014;20 Suppl 6:5-10. [CrossRef]

12. Winston DJ, Limaye AP, Pelletier S, Safdar N, Morris MI, Meneses $\mathrm{K}$, et al. Randomized, double-blind trial of anidulafungin versus fluconazole for prophylaxis of invasive fungal infections in high-risk liver transplant recipients. Am J Transplant 2014;14:2758-64. [CrossRef]

13. Shorr AF, Chung K, Jackson WL, Waterman PE, Kollef MH. Fluconazole prophylaxis in critically ill surgical patients: a meta-analysis. Crit Care Med 2005;33:1928-36. [CrossRef] 